\title{
Static and impact-dynamic characterization of multiphase TRIP steels
}

\author{
Joost Van Slycken ${ }^{1, \text { a }}$, Jeremie Bouquerel ${ }^{2, \mathrm{~b}}$, Patricia Verleysen ${ }^{1, c}$, \\ Kim Verbeken ${ }^{2, d}$, Joris Degrieck ${ }^{1, e}$ and Yvan Houbaert ${ }^{2, f}$ \\ ${ }^{1}$ Department of Materials Science and Engineering, Ghent University, St.-Pietersnieuwstraat 41, \\ Ghent, Belgium \\ ${ }^{2}$ Department of Materials Science and Engineering, Ghent University, Technologiepark 903, Ghent \\ (Zwijnaarde), Belgium \\ a Joost.VanSlycken@UGent.be, ${ }^{b}$ Jeremie.Bouquerel@UGent.be, ${ }^{\mathrm{C}} \mathrm{P}$ atricia.Verleysen@UGent.be, \\ dKim.Verbeken@UGent.be, boris.Degrieck@UGent.be, 'van.Houbaert@UGent.be
}

Keywords: High strain rate behavior, split Hopkinson bar, TRIP steel, elevated test temperatures, interrupted experiments, adiabatic heating, EBSD

\begin{abstract}
In this study, results are presented of an extensive experimental program to investigate the strain rate dependent mechanical properties of various Transformation Induced Plasticity (TRIP) steel grades. A split Hopkinson tensile bar setup was used for the high strain rate experiments and microstructural observation techniques such as LOM, SEM and EBSD revealed the mechanisms governing the observed behavior. With elevated testing temperatures and interrupted tensile experiments the material behavior and the austenite to martensite transformation is investigated. In dynamic conditions, the strain rate has limited influence on the material properties. Yet an important increase is noticed when comparing static to dynamic conditions. The differences in strength, elongation and energy absorption levels observed between the investigated materials can be attributed to their chemical composition. Adiabatic heating during high strain rate deformation tends to slow down the strain induced martensitic deformation. The elongation of the ferritic and austenite constituents is found to be strain rate dependent and the strain induced martensitic transformation occurs gradually in the material.
\end{abstract}

\section{Introduction}

In the automotive industry a lot of effort is put into the development of lightweight car body structures while maintaining crash performance. Therefore complex multiphase steel grades have been developed with exceptional mechanical properties: they combine high strength values with an excellent ductility, resulting in a high energy absorption potential. A key member of this new generation steel grades is the TRansformation Induced Plasticity (TRIP) steel [1]. The excellent properties of this steel grade result from the complex synergy between the different phases in the steel grade and from the transformation of metastable retained austenite into martensite during deformation [2].

To guarantee a controlled dissipation of the energy released during a crash, high strain rate testing of steels is important. The determination of the material properties over a large range of strain rates is thus a key step towards the accurate understanding of vehicle crash tests and of several high speed forming processes. This can for example be done by making advanced use of a split Hopkinson setup [3] and by combining a wide array of experiments with numerical modeling to study the high strain rate behavior of materials.

In this study, results are presented of an extensive experimental program to investigate the strain rate dependent mechanical properties of various TRIP steel grades. Static and dynamic experiments, with strain rates varying from 500 to $2000 \mathrm{~s}^{-1}$, are performed on a wide array of TRIP steel grades with varying alloying elements (Al, Si, P). Several microstructural observation techniques such as LOM, SEM and EBSD are used to reveal the mechanisms governing the observed high strain rate behavior. Special attention is paid to the temperature dependent behavior of TRIP steels. The effect 
of the quasi-adiabatic temperature rise during dynamic deformation and elevated testing temperatures on the material behavior and on the austenite to martensite transformation is investigated.

\section{Experimental setup}

(Quasi-) static behavior. To characterize the (quasi-) static behavior of the investigated materials standard tensile tests are carried out using a screw driven electromechanical Instron type 5569. The tensile tests are performed according to the European standard specifications EN 10002-1:2001and a conventional A80 tensile specimen is used. The tensile tests are carried out with an initial strain rate of $5.610^{-4} \mathrm{~s}^{-1}$, which is increased to $5.610^{-3} \mathrm{~s}^{-1}$ at $3.4 \%$ of deformation. An Instron 2630-118 extensometer with a gauge length of $80 \mathrm{~mm}$ is used to assess the specimen elongation.

Elevated temperature tests are performed at a constant strain rate of $5.610^{-4} \mathrm{~s}^{-1}$. The specimens are put into a specially designed cell filled with anti-freeze liquid with temperatures ranging from -20 to $100^{\circ} \mathrm{C}$.

Impact-dynamic behavior. In order to obtain an in-depth understanding of the behavior of TRIP sheet steels an array of purpose-designed high strain rate experimental setups based on the Hopkinson principle is used [4]. The overall material characterization is performed on the high strain rate Split Hopkinson Tensile Bar (SHTB) setup available at Ghent University (Fig. 1).

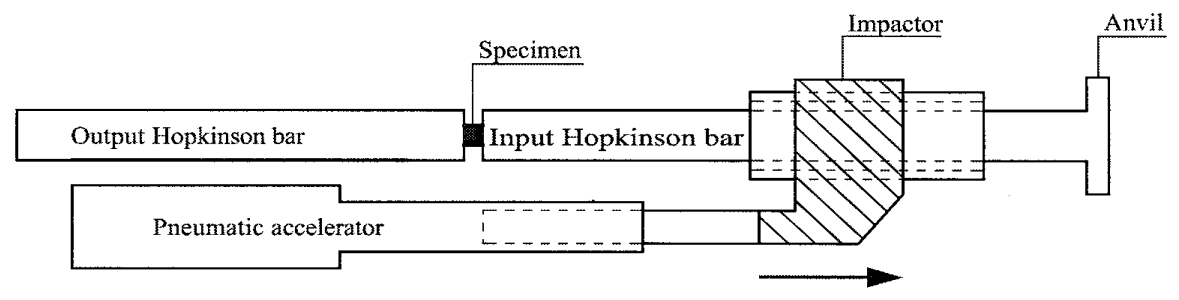

Figure 1: Schematic representation of the Split Hopkinson Tensile Bar setup

The setup mainly consists of two bars: an input and an output bar between which a specimen of the test material is attached. A tensile wave is produced by an impactor that is accelerated towards the anvil of the input bar. After the impact, this incident wave travels along the input bar towards the specimen where it interacts with the sample and is partly reflected back into the input bar. The other part, the transmitted wave, travels along the output bar. The strain histories of the different waves (incident $\varepsilon_{\mathrm{i}}(\mathrm{t})$, reflected $\varepsilon_{\mathrm{r}}(\mathrm{t})$ and transmitted wave $\varepsilon_{\mathrm{t}}(\mathrm{t})$ ) are recorded by means of strain gauges mounted on both bars. By adjusting the impact speed of the impactor, the strain rate can be varied. According to the one-dimensional wave theory and the assumption of a uni-axial and homogeneous stress and strain in the specimen, the stress, strain and strain rate in the specimen can be resp. written as follows [5]:

$$
\sigma(\mathrm{t})=\frac{\mathrm{E}_{\mathrm{b}} \mathrm{A}_{\mathrm{b}}}{\mathrm{A}_{\mathrm{s}}} \varepsilon_{\mathrm{t}}(\mathrm{t}), \quad \varepsilon(\mathrm{t})=\frac{\mathrm{U}_{\mathrm{ob}}-\mathrm{U}_{\mathrm{ib}}}{\mathrm{L}_{\mathrm{s}}}=-\frac{2 \mathrm{C}_{\mathrm{b}}}{\mathrm{L}_{\mathrm{s}}} \int_{0}^{\mathrm{t}} \varepsilon_{\mathrm{r}}(\tau) \mathrm{d} \tau, \quad \dot{\varepsilon}(\mathrm{t})=\frac{\mathrm{V}_{\mathrm{ob}}-\mathrm{V}_{\mathrm{ib}}}{\mathrm{L}_{\mathrm{s}}}=-\frac{2 \mathrm{C}_{\mathrm{b}}}{\mathrm{L}_{\mathrm{s}}} \varepsilon_{\mathrm{r}}(\mathrm{t})
$$

Where, $A_{s}$ and $L_{s}$ are the cross-sectional area and the length of the testing region of the specimen, respectively. $C_{b}$ is the one-dimensional elastic stress wave velocity in the input/output bar material, $A_{b}$ is the cross-section area and $E_{b}$ is Young's modulus of the input/output bar. $U_{i b}$ and $U_{o b}$ are the displacements of the specimen/input bar interface and the specimen/output bar interface, respectively. $\mathrm{V}_{\mathrm{ib}}$ and $\mathrm{V}_{\mathrm{ob}}$ are the corresponding interface velocities. An extensive discussion and several critical remarks on high strain rate testing can be found in [6].

Based on this existing split Hopkinson tensile bar technique, more advanced tensile testing methods are developed by altering some testing features. Tensile test at elevated temperatures (20$80^{\circ} \mathrm{C}$ ) are also performed. This additional temperature rise affects the material behavior and especially the austenite to martensite transformation. With interrupted SHTB experiments the 
specimen is not loaded until fracture but until a certain deformation level. In this way, the microstructure and especially the martensite transformation at different stages of the plastic deformation can be observed. With a succession of short interrupted adiabatic experiments, where the specimen cools down between two succeeding experiments, it is also possible to obtain an approximately isothermal high strain rate experiment [7]. Since the loading time of the specimen is proportional to the length of the impactor, a shorter impactor is necessary. Moreover, in interrupted SHTB experiments the residual stress waves in the Hopkinson bars need to be absorbed to avoid additional loading of the specimen. The use and implementation of these advanced dynamic testing techniques is, however, not straightforward. A detailed description and the experimental implementation of these setups can be found in [6].

\section{Classic dynamic tensile experiments}

The results of the static and high strain rate experiments show clearly that the excellent mechanical properties are not only preserved at higher strain rates, but they are still improved. In dynamic conditions the strain rate has limited influence on the material properties. Yet an important increase is noticed when comparing static to dynamic conditions (Fig. 2a). The differences in strength, elongation and energy absorption levels observed between the investigated materials can be attributed to their chemical composition (Fig. 2b). Silicon contributes to a significant solid solution strengthening and thus high strength levels. TRIP steels mainly alloyed with aluminum on the other hand exhibit lower strength values but higher elongation levels [8].

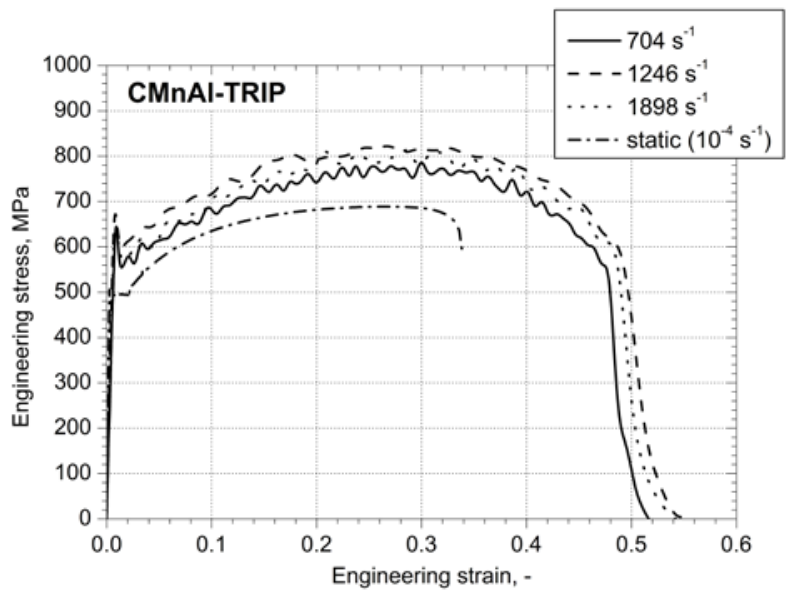

(a)

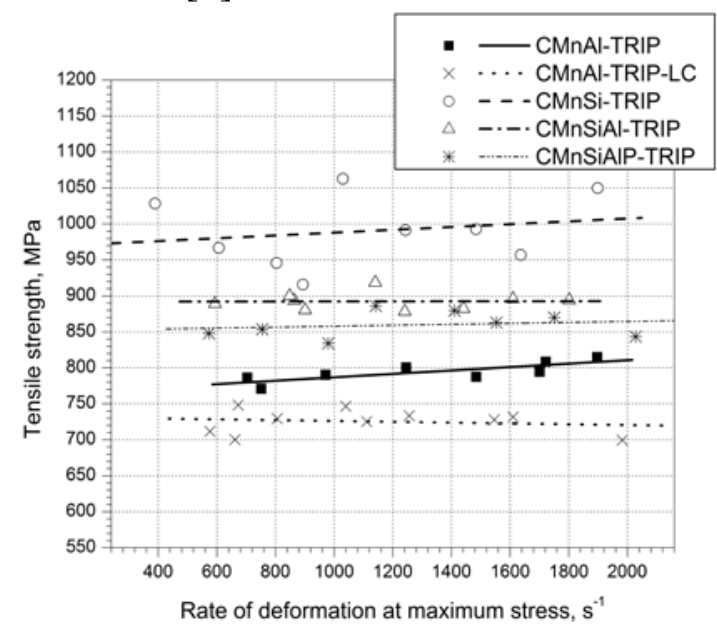

(b)

Figure 2: Representative engineering stress-strain curves for the Al-based TRIP steel grade (CMnAl-TRIP) after static and dynamic deformation. The indicated strain rate is the value reached at maximum stress

\section{Elevated temperatures tensile experiments}

Resulting static true stress-strain curves of a laboratory made SiAl-based TRIP steel (CMnSiAlTRIP) at various temperatures ranging from -20 to $100^{\circ} \mathrm{C}$ are given in Fig. 3a. It can be seen that high testing temperatures are responsible for a decrease in stress level. Moreover, at lower temperatures the curves present a plateau in the beginning of plastic deformation. The retained austenite is less stable at lower temperatures and transforms more easily to martensite. This corresponds to the Greenwood-Johnson effect [9] that results in plastic accommodation carried out at a constant stress. At high strain rate levels higher testing temperatures also cause an important decrease of the stress levels and energy dissipation in the industrial Al-based TRIP steel (Fig. 3b). 


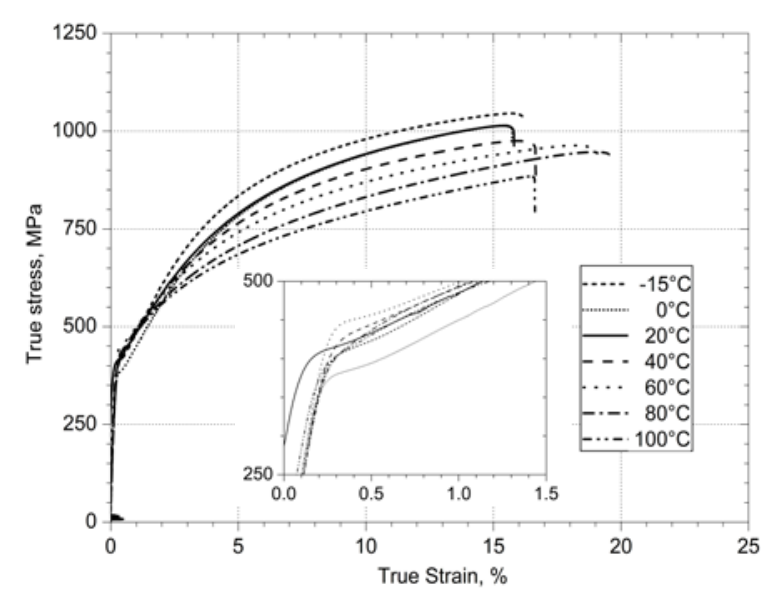

(a)

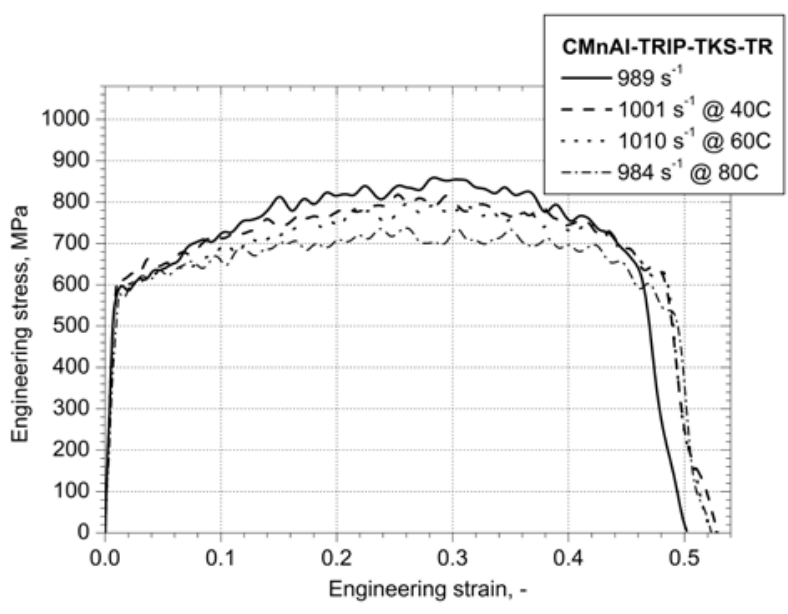

(b)

Figure 3: Influence of the testing temperature on the static true stress-strain curves for a laboratory made SiAl-based TRIP steel (a) and representative high strain rate stress-strain curves for an industrial Al-based TRIP steel (b)

In both static and dynamic cases, TRIP steel presents a pronounced softening with the temperature increase. The uniform deformation slightly decreases with the temperature in the high strain rate tests, whereas it increases for the static tests. Fig. 4a shows, in addition, the evolution of the volume fraction of the transformed austenite (measured by a magnetic saturation technique) with the temperature. A similar trend is observed for static and dynamic deformation. This becomes even clearer when the calculated adiabatic heating is added to the test temperature increase. With higher temperatures, less and less martensite is formed. The austenite is more stable and will gradually transform to martensite close to fracture.

In Fig. 4b the effect of the temperature on the strain induced martensitic transformation is depicted as well as the influence of several alloying elements. An increasing amount of carbon and aluminum will cause a more stable austenite and a more gradual transformation to martensite. The partial replacement of Si by Al causes therefore the decrease of the tensile strength with an increase of the ductility (cf. supra).

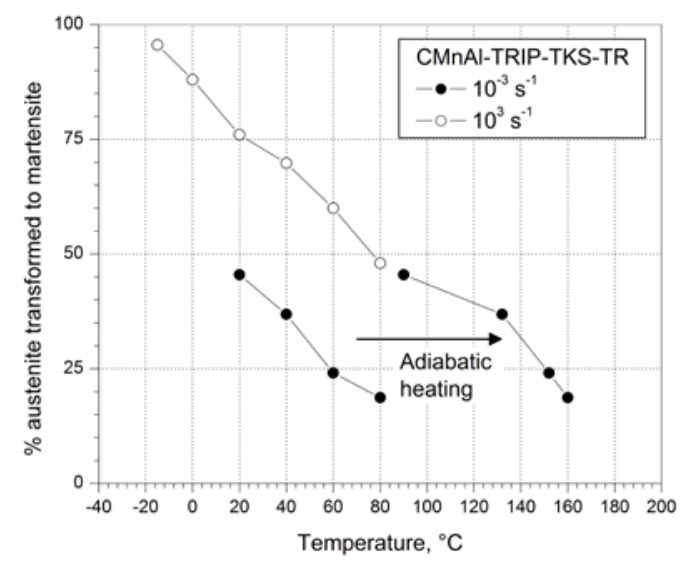

(a)

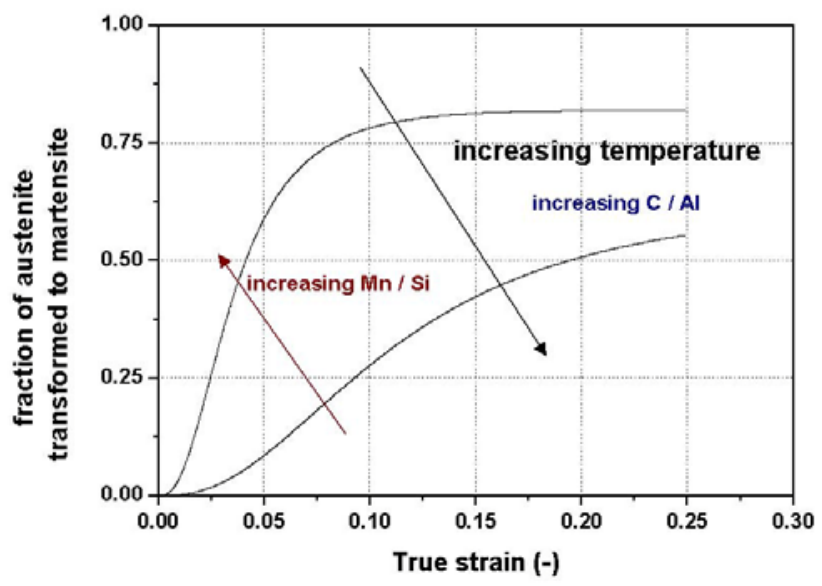

(b)

Figure 4: Influence of the testing temperature on the amount of transformed austenite for an industrial Al-based TRIP steel after static and dynamic deformation (a) and schematic showing the influence of the alloying elements and the testing temperature on the transformation kinetics (b) 


\section{Interrupted tensile experiments}

The dynamic interrupted experiments for an Al-based TRIP steel learn that at $10 \%$ of deformation, $25 \%$ of the retained austenite has already transformed to martensite, whereas after full deformation $50 \%$ of retained austenite is transformed. These observations confirm the results of the Light Optical Microscopy (LOM) and Scanning Electron Microscopy (SEM) evaluation of the microstructure evolution of the statically and dynamically deformed Al-based TRIP steel (Fig. 5). The elongation of the ferritic constituents increases close to the fracture, moreover this elongation is found to be strain rate dependent: higher strain rates result in more deformed grains. The austenite islands adopt a particular behavior as well: for static loading the austenite is slightly deformed and almost fully transformed. Whereas for dynamic loading on the one hand the austenite is highly deformed and transformed close to the fracture and it is slightly deformed and partially transformed away from the fracture on the other hand. The strain induced martensitic transformation occurs gradually in the material, letting time to the austenite to deform and bring more elongation to the TRIP steel. Adiabatic heating during high strain rate deformation tends to slow down the strain induced martensitic deformation [10].

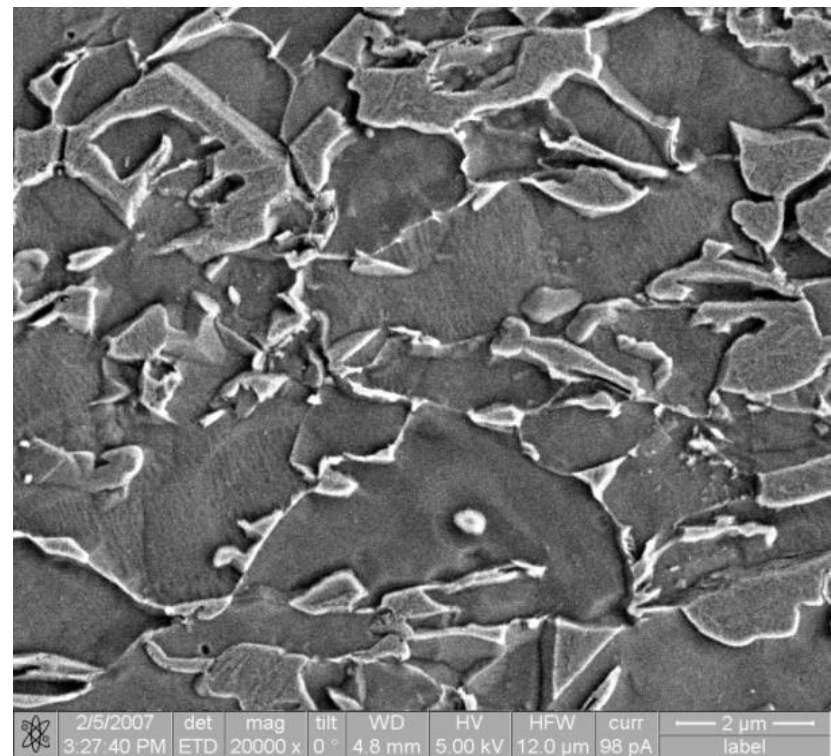

(a)

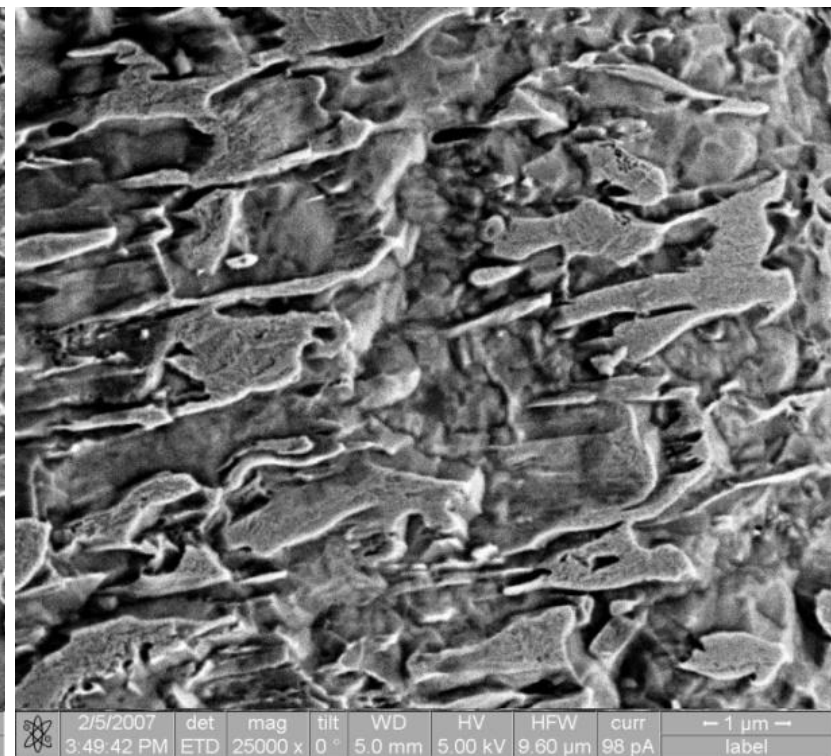

(b)

Figure 5: Microstructure of the Al-based TRIP steel submitted to high strain rate deformation (post testing) (a: away from the fracture, b: close to the fracture tip)

An in-depth characterization of the microstructure can be made by Electron BackScatter Diffraction (EBSD). This technique allows to measure crystallographic orientations, distinguish between different phases and to determine local orientation gradients. The latter can be based on the calculation of the Kernel Average Misorientation (KAM), as was for example demonstrated by Petrov et al. [11]. Figure 6 makes a comparison between the KAM maps for an Al-based TRIP steel that was deformed to different elongations in different modes. This figure shows that the plastic strain is initially localized in the bcc phase near the phase boundaries. With increasing static deformation, the bcc phase accumulates the main part of the deformation whereas the retained austenite remains largely undeformed yet transforms to martensite. After dynamic deformation, the ferritic grains display even bigger orientation gradients, although it must also be pointed out that a higher degree of deformation is reached in the dynamic case. More details on the effect of static and dynamic deformation on the microstructure and texture of these steels are presented elsewhere in this volume [11]. 


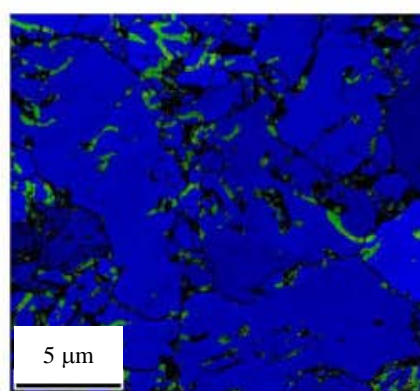

$10 \%$ - static

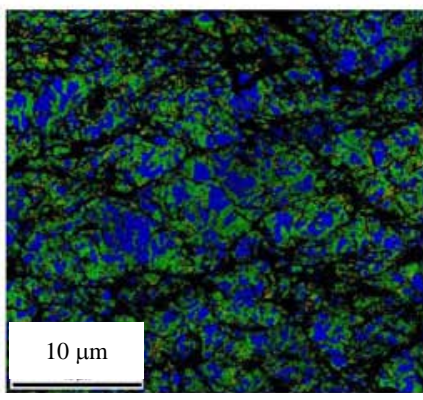

$30 \%$ - static

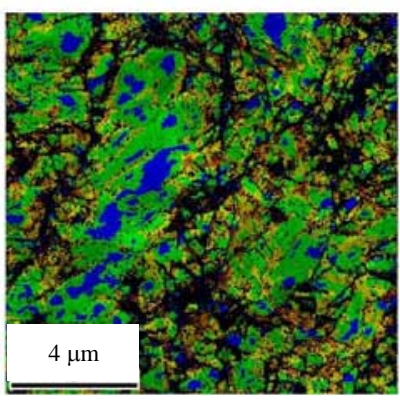

45\%- dynamic

\section{CMnAI TRIP}

Color Coded Man Tvoe

Kernel Average Misorientation

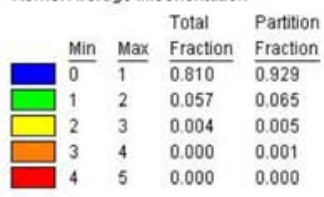

Figure 6: KAM maps of an Al-based TRIP steel during a tensile test with static elongations of $10 \%$ and $30 \%$, and a dynamic elongation of $45 \%$ [11]

\section{Summary}

Results are presented of an extensive experimental program to investigate the strain rate dependent mechanical properties of various TRIP steel grades. With static and dynamic experiments at elevated temperatures and interrupted experiments the material behavior and the austenite to martensite transformation is investigated. In dynamic conditions the strain rate has limited influence on the material properties. Yet an important increase is noticed when comparing static to dynamic conditions. The differences in strength, elongation and energy levels observed between the materials can be attributed to their chemical composition. An increasing amount of carbon and aluminum stabilizes the austenite and causes a more gradual transformation. Adiabatic heating during dynamic deformation slows down the transformation. The elongation of the ferrite and austenite constituents is found to be strain rate dependent and the martensitic transformation occurs gradually in the material. The ferrite phase is mainly responsible for the deformation whereas the retained austenite remains largely undeformed yet transforms to martensite. This causes locally strengthened zones in the ferrite matrix and gives rise to the composite-like strengthening behavior of TRIP steels.

\section{References}

[1] V. Zackay, E. Parker, D. Fahr and R. Bush: Transactions ASM Vol. 60 (1967), p. 252

[2] P.Jacques, J. Ladriere and F. Delannay: Metall Mater Trans A Vol. 32 (2001), p. 2759

[3] B. Hopkinson: Phil. Trans. A Vol. 213 (1914), p. 437

[4] J. Van Slycken, J. Bouquerel, P. Verleysen, K. Verbeken, J. Degrieck and Y. Houbaert: submitted to Journal de Physique IV (2009)

[5] H. Kolsky: Proc. Phys. Soc. Vol. 62 (1949), p. 676

[6] J. Van Slycken: Advanced Use of a Split Hopkinson Bar Setup - Application to TRIP Steels (PhD thesis Ghent University, Belgium 2008).

[7] S. Nemat-Nasser, J.B. Isaacs and J.E. Starett: Proc. R. Soc. London A Vol. 435 (1991), p. 371

[8] J. Van Slycken, P. Verleysen, J. Degrieck, J. Bouquerel and B.C. De Cooman: Met. Mater. Int. Vol. 13 (2007), p. 93

[9] G.W. Greenwood and R.H. Johnson: Proc. Roy. Soc. London A Vol. 283 (1965), p. 403

[10] Bouquerel J., Verbeken K., Krizan D., Barbe L., Verleysen P. and Houbaert Y., Steel research Int, 79 (2008), 2-10.

[11] R. Petrov, K. Verbeken, J. Bouquerel P. Verleysen, L. Kestens and Y. Houbaert: Materials Science Forum, Proceedings Thermec 2009, accepted 